\title{
Acquired Hemophilia A Associated with Venous Thrombosis and Very High Inhibitor Titer: A Challenging Scenario
}

\author{
Napolitano $\mathbf{M}^{1 *}$, Sardo $\mathbf{M}^{1}$, LoCoco $\mathrm{L}^{1}$, Raso $\mathbf{S}^{2}$, \\ Mansueto $\mathrm{MF}^{1}$, Mancuso $\mathrm{S}^{\mathbf{1}}$ and Siragusa $\mathrm{S}^{1}$ \\ ${ }^{1}$ Hematology Unit and Reference Regional Center for \\ Thrombosis and Hemostasis, Department of Health \\ Promotion, Mother and Child Care, Internal Medicine \\ and Medical Specialties, University of Palermo, Italy \\ ${ }^{2}$ Department of Surgical, Oncological and Stomatological \\ Disciplines, University of Palermo, Italy \\ *Corresponding author: Mariasanta Napolitano, \\ Hematology Unit and Reference Regional Center for \\ Thrombosis and Hemostasis, Department of Health \\ Promotion, Mother and Child Care, Internal Medicine \\ and Medical Specialties, University of Palermo, Italy
}

Received: September 21, 2019; Accepted: November 19, 2019; Published: November 26, 2019

\begin{abstract}
Acquired Hemophilia (AH) poses several challenges to clinicians due to potential delays in diagnosis, based on a high index of suspicion, and a high risk of limb and life-threatening bleeding. We here report a case of $\mathrm{AH}$ with extremely high inhibitor titer (up to $1200 \mathrm{BU}$ ) in a patient who also developed venous thrombosis requiring anticoagulant treatment after prolonged immobilization for femur fracture. Multiple lines of immunosuppressive treatment were needed to achieve inhibitor eradication, probably due to the extremely high inhibitor titer, bleeding management also required several lines of treatment with bypassing agents. Bleeding treatment was here monitored by global hemostatic assays. Management of $\mathrm{AH}$ in a reference center allowed to achieve complete remission even in a very intricate condition.
\end{abstract}

\section{Case Report}

Acquired Hemophilia (AH) A is characterized by hemorrhagic manifestations due to the formation of antibodies against human FVIII [1]. The incidence of AH is $1.4 /$ million people/year, idiopathic cases most commonly occur in elderly patients of both sexes [2,3]. Bleeding in $\mathrm{AH}$ is severe and unexpected; it occurs spontaneously or after trauma [2]. The treatment of AH is currently based on results from registries, derived from the experience of single centers. The treatment goals are both controlling and preventing bleeding using bypassing agents and inhibitor eradication with immunosuppressive therapy [3-8]. We here report the unusual case of a patient with $\mathrm{AH}$ and extremely high inhibitor titer who developed venous thrombosis during treatment.

A 69-year-old 54-kg man with diabetes mellitus and rheumatic polymyalgia was transferred to our unit for transfusion-dependent anemia and persistently prolonged Activated Partial Thromboplastin Time (APTT). The patient was first admitted to a psychiatric unit for a reduction in his mood and attempted suicide; a fracture of the left femur secondary to an accidental fall at home was diagnosed. In consideration of the time since the fall, the patient was managed with conservative therapy with complete immobilization and prophylaxis of venous thromboembolism with Low Molecular Weight Heparin (LMWH). During rehabilitative therapy, painful chest wall and right lower limb muscle hematomas occurred associated with progressive anemia requiring transfusion. The patient was then transferred to an internal medical unit after a CT scan detected active bleeding in the thoracic muscle associated with prolonged APTT for which LMWH was discontinued and fresh frozen plasma administered. He was admitted to our unit 12 days after the first detection of prolonged APTT. The patient presented poor general clinical conditions with a high thrombotic risk due to immobilization for the fracture. A clinical exam revealed a large hematoma of the lower and upper limbs and back. His blood count showed severe anemia. Isolated prolonged APTT (106 seconds) was confirmed. AH was suspected, clotting FVIII activity (FVIII:c) and inhibitor to FVIII were tested, and a diagnostic work-up was started including total body CT and PET scans to rule out any underlying diseases. $\mathrm{AH}$ was diagnosed with undetectable FVIII:c and an extremely high titer inhibitor to FVIII (825 BU/ml). Prothrombin time, fibrinogen levels, antithrombin, von Willebrand Factor, coagulation Factor IX, coagulation Factor XIII were all normal. A CT scan with contrast showed a hematoma of the left lateral chest wall $(12 \times 7 \times 5 \mathrm{~cm})$ and an increased bone density area of the left fifth rib. The results of a PET scan were unremarkable.

The treatment schedules for bleeding control and inhibitor eradication are summarized in (Table 1).

The initial treatment of the bleeding was based on Activated Prothrombin Complex Concentrate (APCC), and prednisone was chosen as a single agent immunosuppressant. On day 4 of therapy, APTT was 106 seconds, FVIII:c was $<0.1 \%$, inhibitor was $880 \mathrm{BU} /$ $\mathrm{ml}$, and hemoglobin was persistently low, requiring daily Red Blood Cell (RBC) transfusions. After ruling out concomitant hemolysis and complement activation as a potential contributing cause of anemia, alternate treatment with recombinant activated FVII (rFVIIa) every 6 hours and Prothrombin Complex Concentrate (PCC) was then started. On day 9, due to pain and edema of the right arm at the site of the central line insertion, a color Doppler ultrasound was performed with evidence of partial brachial Vein Thrombosis (VT). Treatment with LMWH at an intermediate dose due to bleeding risk was then contemporary administered. On day 13, due to persistently high inhibitor titer and daily RBC transfusion requirements, treatment with APCC (FEIBA) for bleeding control was administered again with poor results. On day 15, second-line immunosuppressive therapy with anti-CD20 (Rituximab, $375 \mathrm{mg} / \mathrm{m}^{2}$ weekly for 4 weeks) was started. On day 21 , a CT scan was repeated for abdominal and
Ann Hematol Oncol - Volume 6 Issue 10 - 2019

ISSN : 2375-7965 | www.austinpublishing group.com

Napolitano et al. () All rights are reserved
Citation: Napolitano M, Sardo M, LoCoco L, Raso S, Mansueto MF, Mancuso S, et al. Acquired Hemophilia A Associated with Venous Thrombosis and Very High Inhibitor Titer: A Challenging Scenario. Ann Hematol Oncol. 2019; 6(10): 1273. 
Table 1: Treatment and timing schedules.

\begin{tabular}{|c|c|c|c|c|c|c|}
\hline & Dose & Timing & APTT (seconds) & $\begin{array}{c}\text { Mean Hb level, } \\
\text { g/dl }\end{array}$ & FVIII level (inhibitor) & Days after admission \\
\hline \multicolumn{7}{|l|}{ Treatment of bleeding } \\
\hline APCC & $60 \mathrm{U} / \mathrm{Kg} /$ day & Every 8 hours for 3 days & 106 & 7 & $<0.11 \%(880 \mathrm{BU})$ & 1 \\
\hline rFVIIa & $60 \mathrm{mcg} / \mathrm{kg}$ & Every 6 hours for 10 days & $>120$ & 8.5 & NP & 4 \\
\hline PCC & $1000 \mathrm{IU}$ & $\begin{array}{c}\text { Every } 12 \text { hours for } 10 \\
\text { days }\end{array}$ & $>120$ & 8.5 & NP & 4 \\
\hline APCC & 92 U/Kg/day & Every 8 hours for 12 days & $>120$ & 7.2 & NP & 13 \\
\hline PCC & $500 \mathrm{IU}$ & $\begin{array}{c}\text { Every } 12 \text { hours for } 12 \\
\text { days }\end{array}$ & $>120$ & 7.2 & NP & 13 \\
\hline rpFVIII & $100 \mathrm{U} / \mathrm{kg}$ & Twice a day for 1 day & $>120$ & 7 & $<0.1 \%(1246 \mathrm{BU})$ & 26 \\
\hline rpFVIII & $200 \mathrm{U} / \mathrm{kg}$ & Once a day for 1 day & $>120$ & 7 & $0 \%$ & 27 \\
\hline rFVIIa & $90 \mathrm{mcg} / \mathrm{kg}$ & $\begin{array}{l}\text { Every } 3 \text { hours until } \\
\text { discharge }\end{array}$ & $>120$ & 8.4 & NP & 28 \\
\hline \multicolumn{7}{|c|}{ Immunosuppressive therapy } \\
\hline Prednisone & $1 \mathrm{mg} / \mathrm{kg} /$ day & Twice a day for 15 days & 106 & 7 & $<0.1 \%(880 \mathrm{BU})$ & 1 \\
\hline Rituximab & 375 mg/mq & Weekly for 4 weeks & $>120$ & 7.2 & NP & 15 \\
\hline Cyclophosphamide & $2 \mathrm{mg} / \mathrm{kg}$ & Every day & 82 & 10.4 & $0.1 \%$ & 35 \\
\hline \multicolumn{7}{|l|}{ Anticoagulant therapy } \\
\hline LMWH & $5700 \mathrm{IU}$ & Every 24 hours & $>120$ & 8 & NP & 9 \\
\hline
\end{tabular}

lower limb pain, and muscle hematomas in the rectus abdominis, in the left and right gluteus, and in the left femoral rectus were detected. On day 26, due to the lack of clinical and laboratory response, recombinant porcine FVIII, rpFVIII (Obizur) was administered at an initial dosage of $100 \mathrm{U} / \mathrm{Kg}$ (Table 1). At the post-therapy control, FVIII:c was undetectable and the administration of Obizur 100 U/kg was required 6 hours after the first infusion. The next day, a third administration of Obizur at an increased dose of $200 \mathrm{U} / \mathrm{kg}$ was attempted without any response. High titer antibody $(35 \mathrm{BU} / \mathrm{ml})$ against rpFVIII was detected and treatment discontinued. Taking into account the partial response to the previous rFVIIa treatment, rFVIIa therapy was restarted at a standard dose of $90 \mathrm{mcg} / \mathrm{Kg}$ every 3 hours. On day 35, cyclophosphamide as an immunosuppressive agent was added as a persistent high titer inhibitor. On day 57, the patient was transferred to the Department of Rehabilitation Medicine in stable clinical condition and with no active bleeding (APTT was 82 seconds, hemoglobin $10.4 \mathrm{~g} / \mathrm{dl}$, and FVIII activity $0.1 \%$ ). A complete brachial vein thrombosis recanalization was confirmed by Doppler, LMWH was given at intermediate dose for a total of four weeks but it was discontinued for one week (from day 21 to day 28) due to the detection of a new onset hematoma. Ninety days after AH diagnosis, APTT results were normal for the first time with normal FVIII levels and undetectable inhibitor. The last available follow-up was at 120 days with persistently normal parameters and no clinical evidence of any other concomitant disease. summarizes results of global hemostatic assays (thromboelastography, TEG) and thrombin generation performed to monitor treatment. These tests were adopted in an effort to evaluate ability to clot formation during treatment within a context where standard tests (aPTT, FVIII:c) could not be of any support and they resulted able to provide a measure of clot formation during time.

To the best of our knowledge, the clinical case reported herein represents the first case of idiopathic $\mathrm{AH}$ with extremely high inhibitor titer (825 BU) ever described $[9,10]$. It also posed several challenges for concomitant symptomatic VT needing anticoagulation. RpFVIII was here ineffective, possibly due to a high titer rpFVIII inhibitor. The response to immunosuppressive therapy was very late and several combined treatments were required for persistent high titer inhibitor with active bleeding. APCC administration was not immediately effective, while rFVIIa was partially effective as a single agent, probably because it was sufficient to compensate for the lack of FVIII activity by generating an amount of activated FX able to guarantee hemostasis via thrombin formation. Femur fracture could have acted in this specific case as a trigger to the immune system for the constant and high inhibitor synthesis from one side and the development of VT on the other side. The management of $\mathrm{AH}$ in highly specialized settings with frequent clinical and laboratory monitoring has contributed to a successful outcome, even in this extremely risky condition.

\section{References}

1. Kruse-Jarres R, Kempton CL, Baudo F, Collins PW, Knoebl P, Leissinger $\mathrm{CA}$, et al. Acquired hemophilia A: Updated review of evidence and treatment guidance. Am J Hematol. 2017; 92: 695-705.

2. Franchini M, Castaman G, Coppola A, Santoro C, Zanon E, Di Minno G, et al. Acquired Inhibitors of clotting factors: AICE recommendations for diagnosis and management. Blood Transfus. 2015; 13: 498-513.

3. Napolitano M, Siragusa S, Mancuso S, Kessler CM. Acquired haemophilia in cancer: A systematic and critical literature review. Haemophilia. 2018; 24: 43-56.

4. Collins PW. Treatment of acquired hemophilia A. J Thromb Haemost. 2007; 5: 893-900.

5. Baudo $F$, Collins $P$, Huth-Kühne $A$, Lévesque $H$, Marco $P$, Nemes $L$, et al. $\mathrm{EACH} 2$ registry contributors: Management of bleeding in acquired hemophilia A: results from the European Acquired Haemophilia $(\mathrm{EACH} 2)$ Registry. Blood. 2012; 120: 39-46.

6. Zanon E, Pasca S, Santoro C, Gamba G, Siragusa SM, Rocino A, et al. Activated prothrombin complex concentrate (FEIBA $\AA$ ) in acquired haemophilia A: a large multicentre Italian study - the FAIR Registry. $\mathrm{Br} \mathrm{J}$ Haematol. 2019; 184: 853-855.

7. Huth-Kühne A, Baudo F, Collins P, Ingerslev J, Kessler CM, Lévesque H, et 
al. International recommendations on the diagnosis and treatment of patients with acquired hemophilia A. Haematologica. 2009; 94: 566-575.

8. Goudemand J. Treatment of bleeding episodes occurring in patients with acquired haemophilia with FEIBA: the French experience. Haemophilia. 2004; 10: 14 .

9. Mannucci PM, Franchini M. Porcine recombinant factor VIII: an additiona weapon to handle anti-factor VIII antibodies. Blood Transfus. 2017; 15: 365368.

10. Tarantino MD, Cuker A, Hardesty B, Roberts JC, Sholzberg M. Recombinant porcine sequence factor VIII (rpFVIII) for acquired haemophilia A: practical clinical experience of its use in seven patients. Haemophilia. 2017; 23: 25-32. 